\title{
SMS-Nachsorge: Sektorenübergreifende Versorgung für Patientinnen mit Bulimia nervosa
}

\author{
${ }^{a}$ Forschungsstelle für Psychotherapie, Universitätsklinikum Heidelberg, \\ ${ }^{\mathrm{b}}$ AHG Psychosomatische Klinik Bad Pyrmont, Deutschland
}

Stephanie Bauer $^{\mathrm{a}}$ Eberhard Okon ${ }^{\mathrm{b}}$ Rolf Meermann ${ }^{\mathrm{b}}$ Hans Kordy ${ }^{\mathrm{a}}$

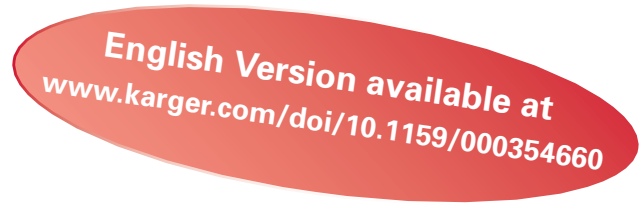

\section{Schlüsselwörter \\ E-Mental-Health · SMS · Nachsorge · Rückfall · Inanspruchnahme}

\section{Zusammenfassung}

Hintergrund: Über den Zusammenhang zwischen der Teilnahme an E-Mental-Health-Interventionen und der Inanspruchnahme regulärer Psychotherapie ist bisher wenig bekannt. In der vorliegenden Studie wurde untersucht, inwiefern ein SMS-basiertes Nachsorgeprogramm mit einem veränderten Inanspruchnahmeverhalten ambulanter Therapie im Anschluss an eine stationäre Behandlung für Essstörungen assoziiert ist. Methode: 165 Patientinnen, die aufgrund einer vollausgeprägten oder subsyndromalen Bulimia nervosa stationär behandelt wurden, wurden randomisiert jeweils einer Kontrollgruppe (KG: treatment as ususal (TAU)) oder Interventionsgruppe (IG: 16-wöchige Nachsorge über SMS zusätzlich zu TAU) zugewiesen. Erfasst wurden die Rückfallrate und die Inanspruchnahme ambulanter Therapie innerhalb des 8-monatigen Follow-up-Zeitraums. Ergebnisse: In der KG wurden signifikant mehr Rückfälle beobachtet als in der IG (KG: 42,0\%; IG: 26,8\%). Vergleichbar viele Teilnehmerinnen nahmen ambulante Therapie in Anspruch (KG: 52,2\%; IG: 53,5\%). Innerhalb der Teilnehmerinnen, die eine ambulante Therapie aufnahmen, unterschieden sich die Rückfallraten von KG und IG nicht signifikant (KG: 38,9\%; IG: 28,9\%). Ein bedeutsamer Unterschied wurde hingegen in der Untergruppe festgestellt, die keine ambulante Therapie nutzte (KG: 45,5\%; IG: 24,2\%). Schlussfolgerung: Die Studie unterstreicht den Bedarf an sektorenübergreifenden Versorgungsangeboten (stationär-ambulant). Die Studie liefert erste Hinweise, dass das SMS-Programm das Inanspruchnahmeverhalten bezüglich ambulanter Therapie positiv beeinflussen könnte. Weitere Studien sind notwendig, um diesen möglichen kausalen Effekt zu untersuchen.

\section{Keywords}

E-mental health - Text messaging - Aftercare - Relapse . Health care utilization

\section{Summary}

Aftercare Based on Text Messaging: Services Across Health Care Sectors for Patients with Bulimia Nervosa Background: Little is known about the relationship between participation in e-mental health interventions and the utilization of regular psychotherapy. The present study investigated to which extent an aftercare program based on text messaging is associated with a change in utilization of outpatient treatment for eating disorders. Method: Following completion of inpatient treatment, 165 patients with full or subthreshold bulimia nervosa were randomized to a control group (CG: treatment as usual (TAU)) or an intervention group (IG: 16 weeks of aftercare via text messaging plus TAU). We assessed rate of relapse and utilization of outpatient treatment within 8 months after discharge. Results: Significantly more relapses occurred in the CG than in the IG (CG: 42.0\%; IG: $26.8 \%$ ). A similar proportion of participants utilized outpatient treatment in both groups (CG: $52.2 \%$; IG: $53.5 \%$ ). In those who did utilize outpatient treatment relapse rates between $\mathrm{CG}$ and IG did not differ significantly (CG: $38.9 \%$; IG: $28.9 \%$ ). However, in participants who did not engage in outpatient treatment a significant difference in the relapse rates between the groups was found (CG: $45.5 \%$; IG: $24.2 \%$ ). Conclusion: The study emphasizes the need for mental health services across different sectors (inpatient-outpatient). The study provides preliminary findings that the text messaging program may positively affect utilization of outpatient treatment. Further research is needed to determine this potentially causal effect of the intervention.

\begin{tabular}{ll}
\hline KARGER & ( ) 2013 S. Karger GmbH, Freiburg \\
Fax +49 761 452 07 14 & Accessible online at: \\
Information@Karger.com & www.karger.com/ver \\
www.karger.com &
\end{tabular}




\section{Einführung}

Informations- und Kommunikationstechnologien (IKT) wecken große Erwartungen für die Gesundheitsversorgung der Zukunft. Zugleich wird die Forderung deutlicher, den konkreten Mehrwert für die Gesundheitsversorgung zu belegen und nachzuweisen, dass der Einsatz von IKT z.B. die Behandlungsmöglichkeiten für konkrete Patientenpopulationen erweitert, den Zugang zu professioneller Hilfe vereinfacht und/oder die Effektivität und Effizienz der Versorgung steigert [WHO, 2010].

Im deutschen Gesundheitssystem stellt die strikte Trennung zwischen dem stationären und ambulanten Versorgungssektor Patienten wie Behandler vor eine große Herausforderung. Jährlich finden ca. 800000 stationäre Aufenthalte in Krankenhäusern (für Psychiatrie, Psychosomatik und Psychotherapie) und Rehabilitationskliniken statt, was sich zu Behandlungskosten von rund 6 Milliarden EUR summiert [Schulz et al., 2008]. Eine effektive Nachsorge im Anschluss an die stationäre Behandlung zur Stabilisierung der erreichten Ergebnisse wird von den Patienten gewünscht und von den Fachkliniken empfohlen [Zielke, 2009]. Katamnestische Daten zeigen, dass eine zeitnahe Nachsorge z.B. in Form einer ambulanten Psychotherapie jedoch häufig nicht gelingt [Kobelt et al., 2004]. Welche Rolle systemimmanente Faktoren (wie z.B. die eingeschränkte Verfügbarkeit von ambulanten Therapieplätzen bzw. lange Wartezeiten) dabei spielen und welchen Anteil Faktoren auf Seiten der Patienten (wie z.B. fehlende Motivation oder die Überzeugung, den Übergang von der Klinik in den Alltag auch allein zu schaffen) dabei haben, ist wenig untersucht. Fest steht jedoch, dass viele Betroffene in der kritischen Zeit nach der Klinikentlassung ohne professionelle Unterstützung bleiben [Zielke, 2009]. In ihrem Krankenhausreport von 2011 berichtet die Barmer GEK [Barmer GEK, 2011], dass innerhalb von 2 Jahren 39\% der Patienten erneut zu einer stationären Behandlung wegen einer psychischen Erkrankung kommen, fast die Hälfte davon bereits in den ersten 3 Monaten nach Entlassung. Auch wenn derzeit noch offen ist, welchen Anteil die Nachsorgelücke daran hat, erscheint eine nahtlos an den Klinikaufenthalt anschließende Unterstützung dringend geraten.

IKT-vermittelte Nachsorgeprogramme nach stationärer Therapie haben sich in einer Reihe von Projekten als vielversprechend erwiesen [z.B. Kordy et al., 2006; Wolf et al., 2006; Golkaramnay et al., 2007, 2009; Bauer et al., 2011a, 2012; Fichter et al., 2012; Ebert et al., 2013]. Die von der Forschungsstelle für Psychotherapie entwickelten Programme «Chat-, E-Mail- und SMS-Brücke» verstehen sich als therapeutische Begleitung beim Übergang von der Klinik in den Alltag- mit oder ohne ambulante Psychotherapie [Golkaramnay et al., 2003]. Teilnehmer der Nachsorgeprogramme können erproben, ob sie mit der IKT-vermittelten Unterstützung alleine zurechtkommen oder die Zeit nutzen und sich in eine poststationäre professionelle ambulante Unterstützung bege- ben. Für die Chat-Brücke zeigte sich, dass die Teilnehmer die sich so ergebende Wahlfreiheit, nach eigenem Bedarf zu entscheiden, zu ihrem Vorteil nutzen konnten: Das größte Rückfallrisiko $(61 \%)$ zeigte sich bei den Patienten, die weder an der Chat-Brücke teilnahmen noch eine ambulante Psychotherapie aufnahmen; das geringste Risiko (16\%) wurde für die Teilnehmer der Chat-Brücke beobachtet, die keine ambulante Psychotherapie aufgenommen hatten. Von denjenigen Patienten, die eine ambulante Psychotherapie aufgenommen hatten, erlebten unter den Teilnehmern der Chat-Brücke 28\% und unter den Nicht-Teilnehmern 37\% einen Rückfall innerhalb von einem Jahr nach Klinikentlassung [Bauer et al., 2011a].

In der Chat-Brücke treffen sich die Teilnehmer mit einem Gruppentherapeuten zu wöchentlichen Gruppensitzungen in einem Chatraum. Die SMS-Brücke basiert hingegen ausschließlich auf dem Konzept des kontinuierlichen supportiven Monitorings und verzichtet auf einen direkten Kontakt zwischen Patient und Therapeut. Daher stellt sich die Frage, ob die für die Chat-Brücke beobachtete verbesserte Passung zwischen individuellem Bedarf und professioneller Unterstützung auch für die weitgehend automatisierte Nachsorge in der SMS-Brücke gelingt.

Die SMS-Brücke wurde von der Forschungsstelle für Psychotherapie und der AHG Psychosomatischen Klinik Bad Pyrmont [Bauer et al., 2003] als ein SMS-vermitteltes Nachsorgeangebot für Patientinnen mit voll- und subsyndromaler Bulimia nervosa entwickelt und in einer vom Bundesministerium für Bildung und Forschung (BMBF) im Schwerpunkt Versorgungsforschung geförderten Wirksamkeitsstudie evaluiert [Bauer et al., 2011b, 2012]. Ausgangspunkt für die Entwicklung stellte die oben genannte Schnittstellenproblematik zwischen stationärer und ambulanter Versorgung dar. Für Bulimia nervosa und verwandte, nicht näher bezeichnete Essstörungen (EDNOS) gilt die kognitive Verhaltenstherapie (KVT) als Behandlung der Wahl [Mitchell et al., 2007; Shapiro et al., 2007; Hay et al., 2009]. Die Remissionsraten liegen jedoch nur bei ca. 40\% [z.B. Agras et al., 2000; Mitchell et al., 2002] und die Rückfallraten nach erfolgreicher Behandlung sind beträchtlich [z.B. Olmsted et al., 1994; Herzog et al., 1999; Keel et al., 2005; Richard et al., 2005]. Das Rückfallrisiko ist unmittelbar nach Erreichen des Zustands der Remission am höchsten und sinkt, wenn es gelingt, diesen Zustand für einige Monate zu stabilisieren [Richard et al., 2005]. Dies unterstreicht die Dringlichkeit einer nahtlosen Unterstützung der Patienten nach Entlassung aus der Fachklinik.

Die Evaluation der SMS-Brücke in einer randomisierten kontrollierten Studie bestätigte die Wirksamkeit des Programms: Während sich bei der Nachuntersuchung 8 Monate nach Klinikentlassung 59\% der Teilnehmerinnen der SMSBrücke im Zustand der Remission befanden, waren dies bei den Nicht-Teilnehmerinnen lediglich 44\% [Bauer et al., 2012]. Auch anhand der sekundären Kriterien (essstörungsbezogene Beeinträchtigung 4 und 8 Monate nach Entlassung [Bauer et 
al., 2011b]) zeigte sich die erwartete Überlegenheit der SMSIntervention gegenüber der Kontrollgruppe.

Ziel der vorliegenden Arbeit war nun die Untersuchung der oben skizzierten Frage, ob und inwiefern die Teilnahme an der SMS-Brücke mit einem veränderten Inanspruchnahmeverhalten bezüglich ambulanter Therapieangebote einhergeht und wie sich das gegebenenfalls auf den Gesundungsverlauf auswirkt.

\section{Methode}

\section{Stichprobe}

Die Stichprobe bestand aus 165 Patientinnen, die randomisiert der Interventionsgruppe $(\mathrm{IG} ; \mathrm{n}=82)$ oder der Kontrollgruppe $(\mathrm{KG} ; \mathrm{n}=83)$ zugewiesen wurden. Die Patientinnen wurden für ca. 8 Wochen (IG: Mittelwert (M) = 55,3 Tage (Standardabweichung (SD) = 9,6); KG: $\mathrm{M}=55,6$ Tage $(\mathrm{SD}=10,3))$ stationär in der AHG Psychosomatischen Klinik Bad Pyrmont behandelt. Etwas mehr als die Hälfte der Patientinnen (IG: 56,1\%; KG: 61,4\%) erfüllte bei Behandlungsbeginn die diagnostischen Kriterien (DSM-IV) einer Bulimia nervosa; die übrigen Patientinnen litten unter einer EDNOS mit deutlich ausgeprägten Essanfällen (definiert als mehr als 1 Essanfall pro Woche für mehr als 4 Wochen). Das Durchschnittsalter lag bei 30 Jahren (IG: $\mathrm{M}=29,87$ ( $\mathrm{SD}=7,91$ ); KG: $\mathrm{M}=30,04$ $(\mathrm{SD}=9,58)$. Eine vergleichbare Anzahl der Patientinnen in beiden Gruppen war alleinstehend (IG: 71,1\%; KG: 67,9\%), verheiratet (IG: 14,5\%; KG: 17,9\%) oder geschieden (IG: 11,8\%; KG: 9,0\%).

\section{Interventionsprogramm}

Das SMS-vermittelte Nachsorgeprogramm [Bauer et al., 2003] zielte auf eine nahtlose Unterstützung der Patientinnen im Anschluss an den Klinikaufenthalt und begann in der Woche der Entlassung aus der Klinik. Die Intervention bestand aus einem wöchentlichen SMS-Austausch zwischen Patientin und Anbieter. Dabei schickte die Patientin einmal pro Woche Informationen zu ihrer Essstörungssymptomatik (d.h. zu ihrer Körperunzufriedenheit sowie zur Häufigkeit von Essanfällen und kompensatorischen Maßnahmen) und erhielt eine auf ihre jeweiligen Angaben abgestimmte Feedbacknachricht. Diese Rückmeldungen, die vom Programm automatisch aus einem Pool von vorformulierten Feedbacknachrichten ausgewählt wurden, hatten im Sinne des supportiven Monitorings das Ziel, günstige Entwicklungen in der Symptomatik positiv zu verstärken. Im Falle von negativen Entwicklungen wurden Strategien zur Verhaltensänderung vorgeschlagen. Die Kommunikation zwischen Patientinnen und Anbieter wurde über ein semiautomatisiertes internetbasiertes Softwareprogramm organisiert, d.h. die vom Programm vorgeschlagene Feedbacknachricht wurde von einer Projektmitarbeiterin auf Plausibilität geprüft und manuell versendet. Die Interventionsdauer betrug in der vorliegenden Studie 16 Wochen.

Es wurden 2 gezielte Notfallvorkehrungen getroffen, um die Sicherheit der Teilnehmerinnen in eventuellen Krisensituationen zu gewährleisten: Zum einen wurden die Teilnehmerinnen instruiert, im Falle einer Krise nicht etwa eine SMS an das Programm zu schicken, sondern den 24-Stunden-Notdienst der Klinik zu kontaktieren, da ihre Nachrichten an das Programm zwar regelmäßig, aber nicht zwingend unmittelbar nach Empfang gelesen würden. Zum anderen benannte jede Teilnehmerin vor Eintritt in die Studie einen sogenannten Notfalltherapeuten in der Nähe ihres Wohnorts, der von der Studienleitung wenn nötig kontaktiert werden könnte (z.B. bei Eingang von SMS mit besorgniserregendem Inhalt). Diese Maßnahme musste jedoch im Verlauf der Studie nicht ergriffen werden. Die Studie wurde von der Ethikkommission der Medizinischen Fakultät Heidelberg genehmigt.

\section{Instrumente}

Die Teilnehmerinnen füllten zu Beginn und Ende der stationären Behandlung sowie 4 und 8 Monate nach Entlassung Fragebögen zu ihrem Gesundheitszustand aus. Da abgesehen von den oben berichteten soziodemographischen Angaben keine Fragebogendaten für die vorliegende Auswertung genutzt wurden, sei für Details zu den eingesetzten Skalen an dieser Stelle auf Bauer et al. [2011b] verwiesen.

Die Rückfallrate wurde über Daten aus katamnestischen Interviews ermittelt, die 8 Monate nach Entlassung telefonisch durchgeführt worden waren. Verwendet wurde die «Longitudinal Interval Follow-up Evaluation» (LIFE) [Keller et al., 1987] mit einer ausführlichen Sektion zu Essstörungen. Das LIFE ist ein halbstrukturiertes Interview, das sich in zahlreichen Studien als reliables und valides Instrument für die retrospektive Erfassung von Symptomverläufen bei psychischen Erkrankungen erwiesen hat [Warshaw et al., 2001]. Dazu bestimmt der Interviewer sogenannte Psychiatric Status Ratings (PSRs) für jede Woche des Beobachtungszeitraums. Der jeweilige PSR-Wert drückt die Krankheitsschwere aus und gibt über 6 Stufen (1-6) an, in welchem Ausmaß die Symptomatik in der jeweiligen Woche die DSM-IV Kriterien der Erkrankung erfüllt. Dabei entsprechen Werte von 6 und 5 einer nach DSMIV voll ausgeprägten Symptomatik, Werte von 4 und 3 einer partiellen Symptomatik und Werte von 2 und 1 stehen für das Nichterfüllen der diagnostischen Kriterien. Die 6-stufige Skala kann zu 3 Schweregraden zusammen gefasst werden (Level 3 = DSM-IV-Kriterien voll erfüllt (PSR 5 oder 6); Level 2 = DSM-IV-Kriterien partiell erfüllt (PSR 4 oder 3); Level 1 = DSM-IV-Kriterien nicht erfüllt (PSR 2 oder 1)). Zusätzlich zu den PSRs wurden von den Interviewern wöchentliche Ratings für die einzelnen Essstörungssymptome vorgenommen (Häufigkeit von Esssanfällen, Erbrechen, Abführmittelgebrauch, etc.). Im Rahmen der vorliegenden Studie wurde vom Vorliegen eines Rückfalls ausgegangen, wenn die PSR-Skala erstmals nach Entlassung aus der Klinik ein Niveau von 3 (d.h. PSR 5 oder 6) erreichte, d.h. eine laut DSM-IV vollausgeprägte Essstörung (Bulimia nervosa oder EDNOS.

Die Inanspruchnahme ambulanter Psychotherapie wurde ebenfalls im Rahmen des LIFE-Interviews erfasst. Die Patientinnen machten Angaben dazu, ob sie seit Entlassung aus der Klinik ambulante Angebote in Anspruch genommen hatten und wenn ja, wie viele Sitzungen sie bis zum Zeitpunkt des Interviews genutzt hatten.

\section{Datenbasis und Auswertung}

Sowohl die Rückfallrate als auch die Inanspruchnahme ambulanter Psychotherapie wurden im Rahmen der LIFE-Interviews erhoben. An den Interviews nahmen 140 der 165 Patientinnen teil (IG: $\mathrm{n}=71$ (87\%); KG: $n=69(83 \%))$. Die Analysen basieren auf dieser Stichprobe. Kaplan-Meier-Überlebenszeitanalysen und Log-rank-Tests wurden für den Vergleich der Gruppen in Hinblick auf die Rückfallrate und Zeit bis zum Rückfall durchgeführt.

\section{Ergebnisse}

\section{Rückfallrate}

Während des 8-monatigen Beobachtungszeitraums wurde bei $26,8 \%$ der Teilnehmerinnen der IG $(n=19)$ und bei $42,0 \%$ der Teilnehmerinnen der KG $(n=29)$ ein Rückfall beobachtet. Während der 4-monatigen SMS-Nachsorge ereigneten sich in der IG 7 Rückfälle (9,9\%). Im selben Zeitraum wurden bereits 21 Rückfälle $(30,4 \%)$ in der KG beobachtet (Tab. 1). Die Überlebenszeitanalyse ergab einen signifikanten Unterschied zwischen IG und KG (Log-rank-Statistik = 4,69, $\mathrm{p}=0,03 ;$ Abb. 1). 
Tab. 1. Anzahl und zeitliche Verteilung der Rückfälle in der Interventions- und Kontrollgruppe

\begin{tabular}{|c|c|c|c|c|c|c|c|c|c|c|c|c|c|c|c|c|c|c|}
\hline Woche & 2 & 4 & 6 & 8 & 9 & 10 & 12 & 14 & 15 & 16 & 17 & 22 & 24 & 25 & 27 & 28 & 29 & 30 \\
\hline $\begin{array}{l}\text { Interventionsgruppe } \\
\qquad(\mathrm{n}=71)\end{array}$ & 1 & 1 & 1 & 1 & & & & & 1 & 2 & 1 & 3 & & 2 & 1 & & 3 & 2 \\
\hline $\begin{array}{l}\text { Kontrollgruppe } \\
\qquad(\mathrm{n}=69)\end{array}$ & 4 & & 2 & 6 & 1 & 1 & 5 & 2 & & & 1 & 1 & 2 & 1 & & 1 & & 2 \\
\hline
\end{tabular}

Abb. 1. Rückfallraten in der Interventionsund Kontrollgruppe (Überlebenskurven).

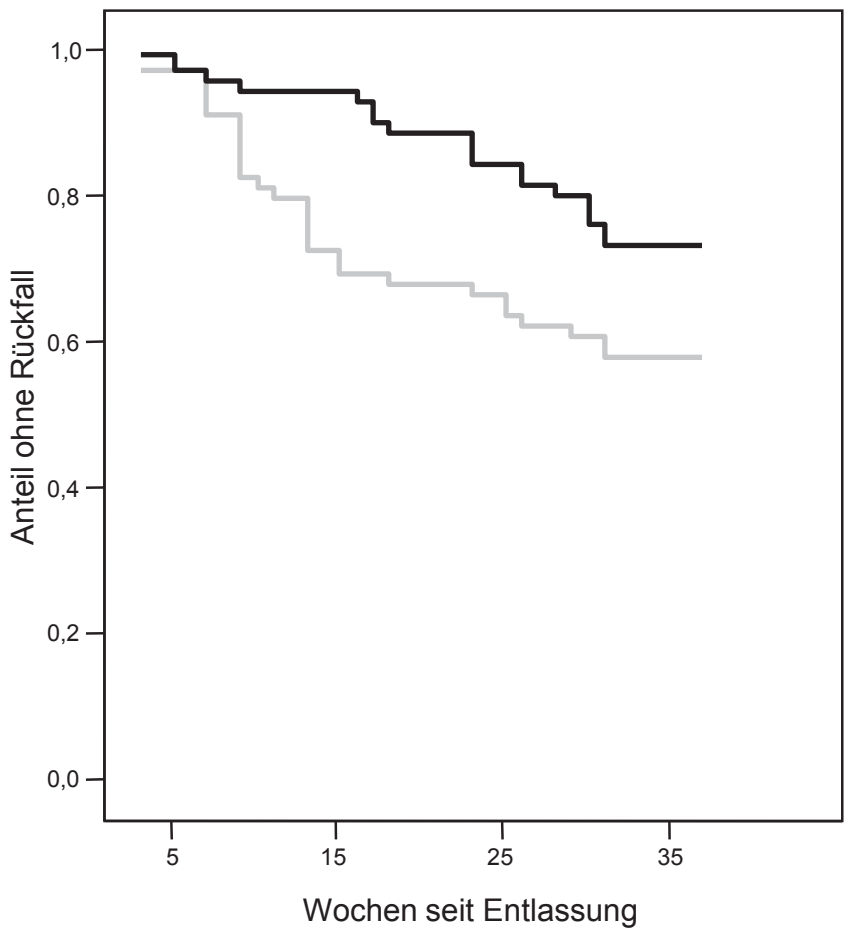

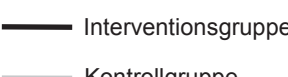

Kontrollgruppe

\section{Inanspruchnahme ambulanter Psychotherapie}

Im Entlassungsfragebogen zu Ende des Klinikaufenthalts bejahten alle Studienteilnehmerinnen die Frage danach, ob sie planen würden, eine ambulante Therapie aufzunehmen. Bezüglich der im Beobachtungszeitraum tatsächlich in Anspruch genommenen Psychotherapie unterschieden sich IG und KG nicht [Bauer et al., 2012]: Der Anteil derjenigen, die eine Behandlung aufnahmen, betrug 53,5\% $(\mathrm{n}=38)$ in der IG und $52,2 \%(\mathrm{n}=36)$ in der $\mathrm{KG}\left(\chi^{2}(1)=0,03, \mathrm{p}=0,87\right)$. Im Mittel nahmen die Teilnehmerinnen der IG 8,52 Sitzungen $(\mathrm{SD}=13,80)$, diejenigen der KG 9,24 $(\mathrm{SD}=15,79)$ in Anspruch $(\mathrm{t}(131)=0,28, \mathrm{p}=0,78)$. Berücksichtigt man nur die Teilgruppen, die eine Behandlung aufgenommen haben, betrugen die Werte 17,03 Sitzungen $(\mathrm{SD}=15,41)$ in der IG und $18,21(\mathrm{SD}=18,18)$ in der KG $(\mathrm{t}(65)=0,29, \mathrm{p}=0,78)$.

\section{Rückfallrate in Teilgruppen mit und ohne ambulante Psychotherapie}

Getrennte Analysen in den Subgruppen mit und ohne ambulante Psychotherapie ergaben folgendes Bild: Bei Teilnehmerinnen, die eine ambulante Behandlung aufnahmen
(IG: $\mathrm{n}=38 ; \mathrm{KG}: \mathrm{n}=36$ ), wurde in der IG eine Rückfallrate von $28,9 \%(\mathrm{n}=11)$ und in der $\mathrm{KG}$ eine Rate von $38,9 \%(\mathrm{n}=$ 14) beobachtet. Die entsprechenden Raten bei Teilnehmerinnen ohne ambulante Therapie (IG: $\mathrm{n}=33$; KG: $\mathrm{n}=33$ ) betrugen $24,2 \%(n=8)$ in der IG und $45,5 \%(n=15)$ in der KG. Die Überlebenskurven sind in Abbildung 2 dargestellt. Die Log-rank-Tests ergaben einen signifikanten Unterschied zwischen IG und KG bei Teilnehmerinnen ohne ambulante Psychotherapie (Log-rank-Statistik $=4,03, \mathrm{p}=0,045$ ), nicht jedoch bei Teilnehmerinnen, die eine ambulante Behandlung aufgenommen hatten (Log-rank-Statistik $=1,10, \mathrm{p}=0,29)$.

\section{Diskussion}

Der Bedarf an Nachsorge nach stationärer Psychotherapie ist weitgehend unbestritten. Die vorliegende Studie liefert einen weiteren Beleg für die Notwendigkeit, Patienten den Zugang zu weitergehenden Unterstützungsangeboten nach Abschluss einer stationären Behandlung zu ermöglichen. Bei fast der Hälfte (42\%) der Patientinnen ist innerhalb von 8 
Abb. 2. Rückfallraten in der Interventionsund Kontrollgruppe mit und ohne ambulante Psychotherapie (Überlebenskurven).

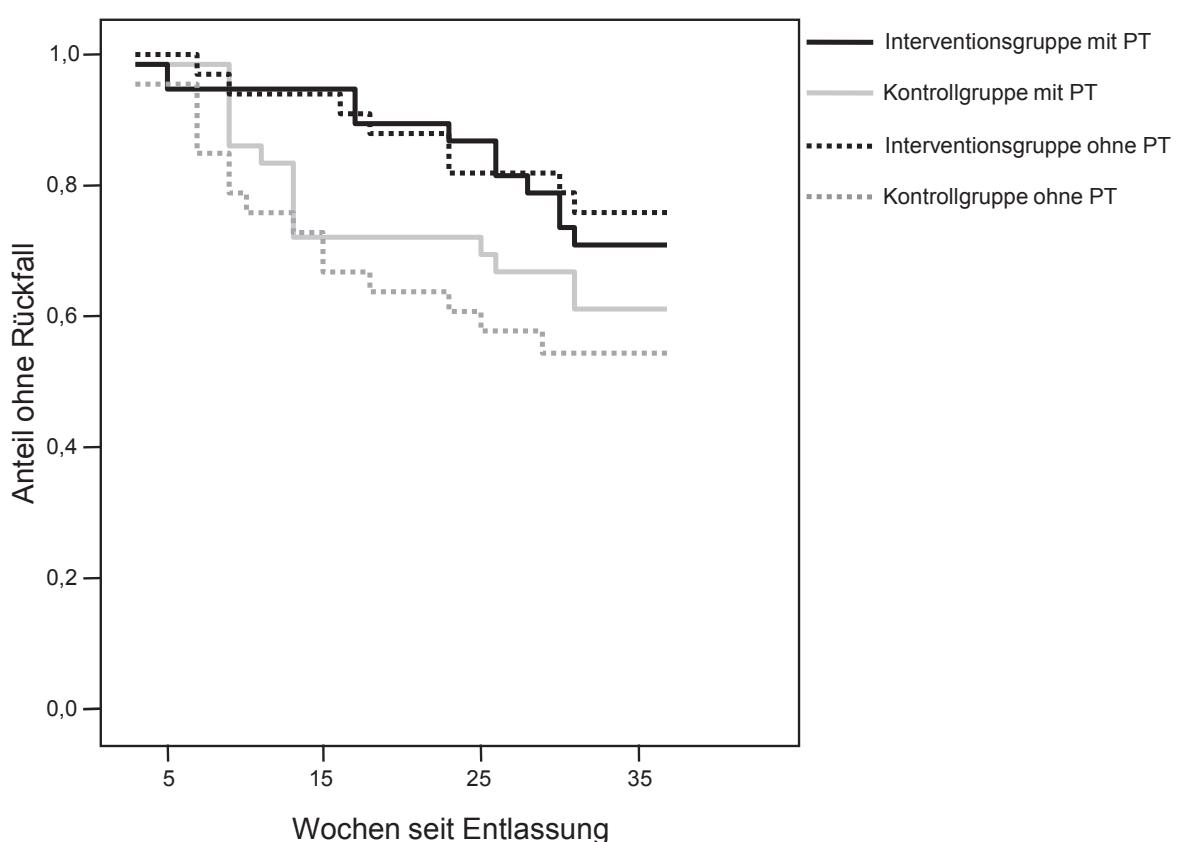

Wochen seit Entlassung
Monaten nach Behandlungsende die vollausgeprägte Essstörung zurückgekehrt, wobei nahezu 75\% der Rückfälle bereits innerhalb der ersten 4 Monate auftraten. Dieses Ergebnis bestätigt Befunde aus anderen Studien, die auf das erhebliche Rückfallrisiko bei Bulimia nervosa, insbesondere kurz nach Erreichen eines remittierten Status, hingewiesen haben [z.B. Olmsted et al., 1994; Richard et al., 2005]. Die SMS-vermittelte Nachsorge konnte die Rückfallrate von 42 auf $27 \%$ senken. Diese durch die longitudinalen LIFE-Daten beschriebene positive Veränderung des Krankheitsverlaufs steht in Einklang mit den Ergebnissen zur Wirksamkeit der SMS-Intervention in den querschnittlichen Prüfungen 4 und 8 Monate nach Ende des Klinikaufenthalts [Bauer et al., 2011b, 2012].

Die Problematik der Trennung von stationärer und ambulanter Versorgung im deutschen Gesundheitssystem zeigt sich auch in der vorliegenden Studie. So nahm nur jede zweite Patientin innerhalb von 8 Monaten nach Ende der Klinikbehandlung eine ambulante Therapie auf, obwohl alle Patientinnen bei Entlassung aus der Klinik dies angestrebt hatten. Rate und Umfang der Inanspruchnahme unterschieden sich nicht zwischen IG und $\mathrm{KG}$; vergleichbar viele Teilnehmerinnen aus beiden Gruppen nahmen im Beobachtungszeitraum eine Therapie sowie im Mittel ähnlich viele Sitzungen in Anspruch. Mit dem SMS-Programm stand den Teilnehmerinnen der IG aber eine weitere Option der Unterstützung zur Verfügung.

Die getrennte Analyse der Rückfallraten in den Teilgruppen mit und ohne ambulante Psychotherapie ergibt ein interessantes Bild: Das geringste Rückfallrisiko zeigte sich für diejenigen Patientinnen, die nur an der SMS-Brücke teilgenommen hatten $(24,2 \%)$ und ein beinahe doppelt so großes für diejenigen, die keinerlei professionelle Nachsorge in Anspruch genommen hatten $(45,5 \%)$. Die Rückfallraten zwischen IG und KG bei Patientinnen, die eine ambulante The- rapie aufnahmen, lagen dazwischen und unterschieden sich nur wenig (28,9\% vs. $38,9 \%$ zugunsten der IG). Eine plausible Möglichkeit der Interpretation stellt die eingangs angesprochene verbesserte Passung zwischen Unterstützungsbedarf und -angebot dar, die sich durch die SMS-vermittelte Nachsorge ergibt. Die Teilnehmerinnen der IG hatten die Option, sich erst dann zur Inanspruchnahme ambulanter Behandlung zu entschließen, wenn sie die SMS-vermittelte automatisierte Unterstützung als nicht (mehr) ausreichend erlebten. Demnach ließe sich vermuten, dass, ähnlich wie bei der ChatBrücke [Bauer et al., 2011a], unterschiedliche Teilpopulationen in IG und KG ambulante Therapie in Anspruch genommen haben. Leider fehlen hierzu Daten zum Entscheidungsprozess, da diese weitergehende Fragestellung erst mit dieser Detailanalyse aufkam. Aufgrund der vorliegenden Ergebnisse erscheint es lohnenswert, den Zusammenhang zwischen der Inanspruchnahme von IKT-vermittelten Angeboten und ambulanter Psychotherapie in zukünftigen Studien systematisch zu untersuchen. Detailliertere Informationen zur Aufnahme (z.B. Motivation, Rahmenbedingungen, Erfahrungen) und Inanspruchnahme (z.B. Informationen zu Beginn und Ende der Behandlung, Sitzungsfrequenz) könnten parallel zur Beobachtung des Symptomverlaufs Aufschluss darüber geben, welche Chancen sich durch eine IKT-vermittelte Nachsorge für eine effiziente, dem individuellen Bedarf entsprechende Gesundheitsversorgung ergeben.

\section{Disclosure Statement}

Die Studie wurde vom BMBF im Programm «Versorgungsforschung» gefördert (01GL0503). Die Autoren erklären, dass keinerlei Interessenskonflikte im Zusammenhang mit der vorliegenden Studie bestehen. 


\section{Literatur}

Agras WS, Walsh T, Fairburn CG, Wilson GT, Kraemer HC: A multicenter comparison of cognitivebehavioral therapy and interpersonal psychotherapy for bulimia nervosa. Arch Gen Psychiatry 2000; 57:459-466.

Barmer GEK (ed): Barmer GEK Report Krankenhaus 2011. Schriftenreihe zur Gesundheitsanalyse, Band 9. St. Augustin, Asgard Verlag, 2011.

-Bauer S, Percevic R, Okon E, Meermann R, Kordy H: Use of text messaging in the aftercare of patients with bulimia nervosa. Eur Eat Dis Rev 2003;11:279-290.

Bauer S, Wolf M, Haug S, Kordy H: The effectiveness of internet chat groups in the relapse prevention after inpatient psychotherapy. Psychother Res 2011a;21:219-226.

Bauer S, Okon E, Meermann R: Nachsorge nach stationärer Psychotherapie für Essstörungen: Wirksamkeit eines SMS-basierten Programms. Psychotherapeut 2011b;56:509-515.

Bauer S, Okon E, Meermann R, Kordy H: Technology-enhanced maintenance of treatment gains for eating disorders: Efficacy of an aftercare intervention delivered via text messaging. J Consult Clin Psychol 2012;80:700-706.

Ebert D, Tarnowski T, Gollwitzer M, Sieland B, Berking M: A transdiagnostic internet-based maintenance treatment enhances the stability of outcome after inpatient cognitive behavioral therapy: a randomized controlled trial. Psychother Psychosom 2013:82:246-256.

- Fichter MM, Quadflieg N, Nisslmüller K, Lindner S, Osen B, Huber T, Wünsch-Leiteritz W: Does internet-based prevention reduce the risk of relapse for anorexia nervosa? Behav Res Ther 2012;50:180-190.

Golkaramnay V, Wangemann T, Dogs J, Dogs P, Kordy H: Neue Brücken für Lücken in der psychotherapeutischen Versorgung durch das Internet: Hoffnungen, Herausforderungen und ein Lösungsansatz. Psychother Psychosom Med Psychol 2003; 53:399-405.
Golkaramnay V, Bauer S, Haug S, Wolf M, Kordy H: The exploration of the effectiveness of group therapy through an internet chat as aftercare: a controlled naturalistic study. Psychother Psychosom 2007; 76:219-225.

Golkaramnay V, Cicholas B, Vogler J: Wirksamkeit einer internetgestützten Nachsorge nach stationärer psychosomatischer Rehabilitation. DRV-Schriften 2009:88: 171-172.

Hay PP, Bacaltchuk J, Stefano S, Kashyap P: Psychological treatments for bulimia nervosa and binging. Cochrane Database Syst Rev 2009;7:CD000562.

Herzog DB, Dorer DJ, Keel PK, Setwyn SE, Ekeblad ER, Flores AT, Greenwood DN, Burwell RA, Keller MB: Recovery and relapse in anorexia and bulimia nervosa: A 7.5-year follow-up study. J Am Acad Child Adolesc Psychiatry 1999;38:289-837.

Keel PK, Dorer DJ, Franko DL, Jackson SC, Herzog DB: Postremission predictors of relapse in women with eating disorders. Am J Psychiatry 2005;62: 2263-2268.

Keller MB, Lavori PW, Friedman B, Nielsen E, Endicott J, McDonald-Scott P, Andreasen NC: The Longitudinal Interval Follow-up Evaluation. A comprehensive method for assessing outcome in prospective longitudinal studies. Arch Gen Psychiatry 1987;44:540-548.

Kobelt A, Nickel L, Grosch E, Lamprecht F, Künsebeck H-W: Inanspruchnahme psychosomatischer Nachsorge nach stationärer Rehabilitation. Psychother Psych Med 2004;54:58-64.

Kordy H, Golkaramnay V, Wolf M, Haug S, Bauer S: Internetchatgruppen in Psychotherapie und Psychosomatik: Akzeptanz und Wirksamkeit einer Internet-Brücke zwischen Fachklinik und Alltag. Psychotherapeut 2006;51:144-153.

Mitchell JE, Halmi K, Wilson GT, Agras WS, Kraemer $\mathrm{H}$, Crow S: A randomized secondary treatment study of women with bulimia nervosa who fail to respond to CBT. Int J Eat Disord 2002;32:271-281.
Mitchell JE, Agras WS, Wonderlich S: Treatment of bulimia nervosa: where are we and where are we going? Int J Eat Dis 2007;40:95-101.

Olmsted MP, Kaplan AS, Rockert W: Rate and prediction of relapse in bulimia nervosa. Am J Psychiatry 1994;151:738-743.

Richard M, Bauer S, Kordy H: Relapse in anorexia and bulimia nervosa. Eur Eat Dis Rev 2005;13:180190.

Schulz H, Barghaan D, Harfst T, et al.: Psychotherapeutische Versorgung. Berlin, Robert Koch Institut, 2008.

Shapiro JR, Berkman ND, Brownley KA, Sedway JA, Lohr KN, Bulik CM: Bulimia nervosa treatment: a systematic review of randomized controlled trials. Int J Eat Disord 2007;40:321-336.

Warshaw MG, Dyck I, Allsworth J, Stout RL, Keller MB: Maintaining reliability in a long-term psychiatric study: an ongoing inter-rater reliability monitoring program using the longitudinal interval followup evaluation. J Psychiatr Res 2001;35:297-305.

Wolf M, Maurer W-J, Dogs P, Kordy H: E-Mail in der Psychotherapie - ein Nachbehandlungsmodell via Electronic Mail für die stationäre Psychotherapie. Psychother Psychosom Med Psychol 2006;56:138146.

World Health Organization (WHO): Telemedicine: Opportunities and Developments in Member States: Report on the 2nd Global Survey on eHealth 2009. Geneva, WHO Press, 2010.

Zielke M: Die psychotherapeutische Ambulanz im Vorfeld und im Nachgang stationärer verhaltensmedizinischer Behandlungen und Rehabilitationsmaßnahmen in der Psychosomatik - Differenzielle Zuweisungen und Krankheitsverläufe. Praxis Klinische Verhaltensmedizin und Rehabilitation 2009 84:84-108. 